\title{
Experimental Analysis of Self-Similarity and Random Cascade Processes: Application to Fully Developed Turbulence Data
}

\author{
A. Arneodo (*), J.F. Muzý and S.G. Roux \\ Centre de Recherche Paul Pascal, avenue Schweitzer, 33600 Pessac, France
}

(Received 4 November 1996, received in final form 19 December 1996, accepted 19 December 1996)

PACS.02.50.-r - Probability theory, stochastic processes, and statistics

PACS.47.27.Gs - Isotropic turbulence; homogeneous turbulence

\begin{abstract}
In the context of fully developed turbulence, Castaing et al. [10] have recently advocated a description of a random cascade process in terms of a kernel $G_{a a^{\prime}}(x)$ that characterizes the nature of the cascade when going from a scale $a^{\prime}$ to a finer scale $a$. We elaborate on a method to estimating, directly from experimental data, the shape of $G_{a a^{\prime}}$ for all scales $a$ and $a^{\prime}$. We apply this method to turbulent velocity data and we show that it provides very instructive informations about the soundness of various phenomenological models for the intermittency character of turbulent flows.
\end{abstract}

It is now well-accepted $[1,2]$ that in the fully developed regime, a turbulent flow is likely to be in a universal state that can be experimentally characterized by statistical quantities such as the scaling exponents $\zeta_{p}$ of the velocity structure functions or the shape of the velocity increment probability density functions (pdf). For more than thirty years, one of the main features recognized experimentally, is the intermittency of small scales [1-4]; this phenomenon manifests in a nonlinear behavior of $\zeta_{p}$ that does not seem to depend on the Reynolds number [5]. This observed violation of the scaling properties predicted by the dimensional theory of Kolmogorov [6] (K41) has been attributed to the large spatial fluctuations of the energy dissipation local rate. Since Richardson's pioneering work [1], further developed by Kolmogorov and Obukhov [4] (KO62), the energy transfer from large to small scales is often seen as a cascade process resulting from strong nonlinear interactions. The notions of "self-similarity", "fractals" and "scale-similarity" introduced by Mandelbrot [7] and Novikov [8] have provided some mathematical framework to a lot of cascade models ranging from the early proposed " $\beta$-model" $[2,3]$ to the more recent "log-Poisson" description of the dissipation field [9].

In a series of papers [10], Castaing and his collaborators, who were mainly interested in the shape of the velocity increment pdf, proposed a description based on a functional equation that relates two scales using a kernel $G$. This description relies upon the ansatz that the pdf $P_{\ell}(\delta v)$ of the velocity increments over a distance $\ell$, can be expressed as a weighted sum of dilated pdfs of increments over a distance $\ell^{\prime}>\ell: P_{\ell}(\delta v)=\int G_{\ell \ell^{\prime}}(\ln r) \frac{1}{r} P_{\ell^{\prime}}\left(\frac{\delta v}{r}\right) \mathrm{d} r=$, where $G_{\ell \ell^{\prime}}$ is a kernel that depends on $\ell$ and $\ell^{\prime}$ only. Indeed most of the well-known cascade models can- be reformulated within this approach $[10 \mathrm{~d}, \mathrm{e}]$. This amounts i) to specify the shape of the kernel

$\left(^{*}\right)$ Author for correspondence (e-mail: arneodo@crpp.u-bordeaux.fr)

(C) Les Éditions de Physique 1997 
$G(x)$ which is determined by the nature of the elementary step in the cascade and ii) to define the way $G_{\ell \ell^{\prime}}$ depends on both $\ell$ and $\ell^{\prime}$.

In this paper, we address the issue of the estimation of $G$ from experimental data. Partial results were obtained by Castaing et al. [10] who succeeded to show that, under some mild hypothesis, the variance of $G$ and its scale behavior could be estimated. Our goal is to show that one can go a step further and extract the entire shape of the Fourier transform of $G$ for any couple of scales. Our method will be tested on various examples of self-similar functions and then applied to turbulent velocity signals.

Let us first generalize the previous ansatz and define the notion of abstract cascade. Usually a cascade process refers to a multiplicative way of spreading mass or energy (i.e. a positive quantity) within smaller and smaller boxes. The cascade is then defined by the way the scales are refined (e.g. divided by a factor two at each step) and the statistics of the multiplicative factors (e.g. log-normal distribution). The limitations of such models are obvious: i) they concern positive fields only and ii) the statistics at fixed analyzing scale does not obviously correspond to the statistics at a fixed construction level of the cascade $[3,11]$. To circumvent these difficulties, we will consider instead cascade models for the construction of the wavelet transform (WT) of the considered field. Actually, wavelet analysis [12] is the general mathematical framework for analyzing signals in both space and scale without loss of information. The WT of a function $f$ is defined by [12]: $T(b, a)=a^{-1} \int \psi\left(\frac{x-b}{a}\right) f(x) \mathrm{d} x$, where $\psi(x)$ is the analyzing wavelet. Let us point out that the increment of $f$ at a point $x_{0}$ over a distance $l$ can be seen as the wavelet coefficient $T\left(x_{0}, l\right)$ using the "poor man's" analyzing wavelet $\psi(x)=\delta(x+1)-\delta(x)$. More generally, $\psi$ is chosen to be well localized not only in direct space but also in Fourier space (the scale $a$ can thus be seen as the inverse of a local frequency). The WT has been advocated as a powerful tool to analyze fractal distributions [13]. It associates to a function on $\mathbb{R}$, its transform defined on $\mathbb{R} \times \mathbb{R}^{+}$and is thus very redundant. To reduce the amount of information to process, one can either work with an orthonormal wavelet basis [12] or restrict ourselves to the modulus maxima of the WT [14].

We can now define an abstract cascade as a process whose WT coefficient pdf at a scale $a$ can be written in terms of the pdf at a coarser scale $a^{\prime}$ as: $\forall a<a^{\prime}<L$ (the largest scale in the problem), $\exists G_{a a^{\prime}}(x)$ such that

$$
P_{a}(T)=\int G_{a a^{\prime}}(x) P_{a^{\prime}}\left(\mathrm{e}^{-x} T\right) \mathrm{e}^{-x} \mathrm{~d} x .
$$

The way of the shape of $G_{a a^{\prime}}(x)$ may depend on the analyzing wavelet $\psi$ and on the fact that one restricts oneself to coefficients on the dyadic grid or at the modulus maxima points is discussed below. The term cascade is used because, from the above definition, one can show that, for any decreasing sequence of scales $\left(a_{1}, \ldots, a_{n}\right)$, one has [10]: $G_{a_{n} a_{1}}=G_{a_{n} a_{n-1}} \otimes \ldots \otimes G_{a_{2} a_{1}}$, where $\otimes$ denotes the convolution product. Referring to the work of Castaing et al. [10], we will say that the cascade is self-similar if there exists a decreasing sequence of scales $\left\{a_{n}\right\}$ such that $G_{a_{n} a_{n-1}}=G$ is independent of $n$. The cascade is said continuously self-similar if there exists a positive, monotonous function $s(a)$, such that $G_{a a^{\prime}}$ depends on $a$ and $a^{\prime}$ only through $s\left(a, a^{\prime}\right)=s(a)-s\left(a^{\prime}\right): G_{a a^{\prime}}(x)=G\left(x, s\left(a, a^{\prime}\right)\right)$. Using Novikov's definition [8], we will say that the cascade is scale-similar if $s\left(a, a^{\prime}\right)=\ln \left(a / a^{\prime}\right)$. By analogy with stochastic processes, if one identifies $t$ with $\ln a$, self-similarity is equivalent to consider processes with independent increments and scale-similarity amounts to impose the process to be stationary.

From a general point of view, given a function $G$, it is not obvious that there exists a process that meets the above requirements and if it is so, that this process be unique. But one can show that most multiplicative cascade models correspond to scale-similar abstract cascades. For example, for a multiplicative cascade defined on an orthonormal wavelet basis [15], 
a wavelet coefficient $C_{\jmath, k}$, at scale $a=2^{-\jmath}$ and position $b=k 2^{-\jmath}$, can be written as $\Pi_{\imath=1}^{3} W_{\imath}$ where $\left[V_{\imath}\right.$ are i.i.d. random variables of given law $\rho$. It is then easy to prove that $P_{\jmath}(C)=$ $\int G(x) P_{\jmath-1}\left(\mathrm{e}^{-x} C\right) \mathrm{e}^{-x} \mathrm{~d} x$ where $G(x)=\mathrm{e}^{x} \rho\left(\mathrm{e}^{x}\right)$ is the pdf of $\ln W$. The multiplicative cascade is therefore a scale-similar cascade. Because the statistics of $W$ can be proved to be independent of the analyzing wavelet [13], the kernel $G$ does not depend on $\psi$. More generally, for self-similar processes, one expects $G_{a a^{\prime}}$ to depend upon the analyzing wavelet $\psi$ only through a multiplicative factor in $s\left(a, a^{\prime}\right)$.

Let us now switch to the numerical estimation of $G$. Let $M(p, a)$ be the characteristic function associated to the logarithm of the wavelet coefficients at scale $a: M(p, a)=$ $\int \mathrm{e}^{\imath p \ln (|T|)} P_{a}(T) \mathrm{d} T$. From equation (1), it is easy to show that $\hat{G}$, the Fourier transform of $G$, satisfies:

$$
M(p, a)=M\left(p, a^{\prime}\right) \hat{G}_{a a^{\prime}}(p) .
$$

From the convolution property of $G$ and the additivity of the function $s$, one can see that the cascade is continuously self-similar if and only if

$$
\hat{G}_{a a^{\prime}}(p)=\hat{G}(p)^{s\left(a, a^{\prime}\right)}
$$

From this equation, one deduces that $\hat{G}$ has to be the characteristic function of an infinitely divisible pdf. Such cascade is referred to as a log-infinitely divisible cascade.

If the characteristic function $M\left(p, a^{\prime}\right)$ does not vanish, one can then estimate $\hat{G}_{a a^{\prime}}(p)$ as the ratio $M(p, a) / M\left(p, a^{\prime}\right)$. Numerically, the real and imaginary parts of this complex quantity are computed separately from the estimates of the mean of $\cos (p \ln |T|)$ and $\sin (p \ln |T|)$ over the WT coefficients at scales $a$ and $a^{\prime}$. To avoid numerical instabilities over a reasonable range of values of $p$, one needs that $M\left(p, a^{\prime}\right)$ remains large as compared to numerical noise. This is practically achieved by restricting our analysis to the WT modulus maxima [13] (instead of using the continuous WT).

Let us illustrate these idea on some simple examples. In Figure 1a, we show the computation of $\hat{G}$ for Brownian motion. If a process is homogeneously scale invariant, the pdf of its WT coefficients satisfies $P_{a}(T)=\left(\frac{a}{a^{\prime}}\right)^{-H} P_{a^{\prime}}\left(\left(\frac{a}{a^{\prime}}\right)^{-H} T\right)$ ( $H$ is the Hölder exponent of the path of the process; $H=1 / 2$ for Brownian motion). A straightforward calculation yields $\hat{G}_{a a^{\prime}}(p)=$ $e^{2 p H \ln \left(a / a^{\prime}\right)}$ which attests that the process is continuously scale-similar: $\hat{G}$ is a pure wave of pulsation $H \ln \left(a / a^{\prime}\right)$. Numerical estimates of the real and imaginary parts of $\hat{G}$ are compared to the above analytical expression for $\log _{2}\left(a^{\prime} / a\right)=6$. One can see that for $p \in[-6,6]$, the agreement is very good. The deviations from the theoretical predictions for larger values of $|p|$ come mainly from finite statistics effects and the rapid decrease of $M\left(p, a^{\prime}\right)$. In Figure $1 \mathrm{~b}$, similar calculations are performed for a Bernoulli measure that is build by dispatching, at each step, the measure on two half-subintervals with multiplicative weights $\mu_{1}=0.6$ and $\mu_{2}=0.4$ respectively. For $a=2^{-n}$ and $a^{\prime}=2^{-m}, \hat{G}_{a a^{\prime}}$ is simply given by the expression: $\hat{G}_{a a^{\prime}}(p)=\left(\frac{\mu_{1}^{2 p}+\mu_{2}^{2 p}}{2}\right)^{n-m}$ One can see that this process is again scale-similar since $G$ depends only on $n-m=\log _{1 / 2}\left(a / a^{\prime}\right)$. One can check in Figure $1 \mathrm{~b}$ that the above expression provides a very good fit of the numerical data obtained using our method.

Recently, there has been a renewal of interest for the so-called log-infinitely divisible distributions [8-10] in the context of fully developed turbulence modelling. A well known example of such distributions is the log-normal model originally introduced by Kolmogorov and Obukhov [4]. Another example comes from the recent works of She et al. [9] and Dubrulle [16a] that show that a cascade model involving log-Poisson statistics provides a remarkable fit to the available experimental data. These two models are scale-similar since the function $s\left(a, a^{\prime}\right)$ can be proved to be $\ln \left(a / a^{\prime}\right)$. In Figure 2, our method for estimating $\hat{G}$ is tested on both these distributions. The models we analyze are a log-normal and a log-Poisson 


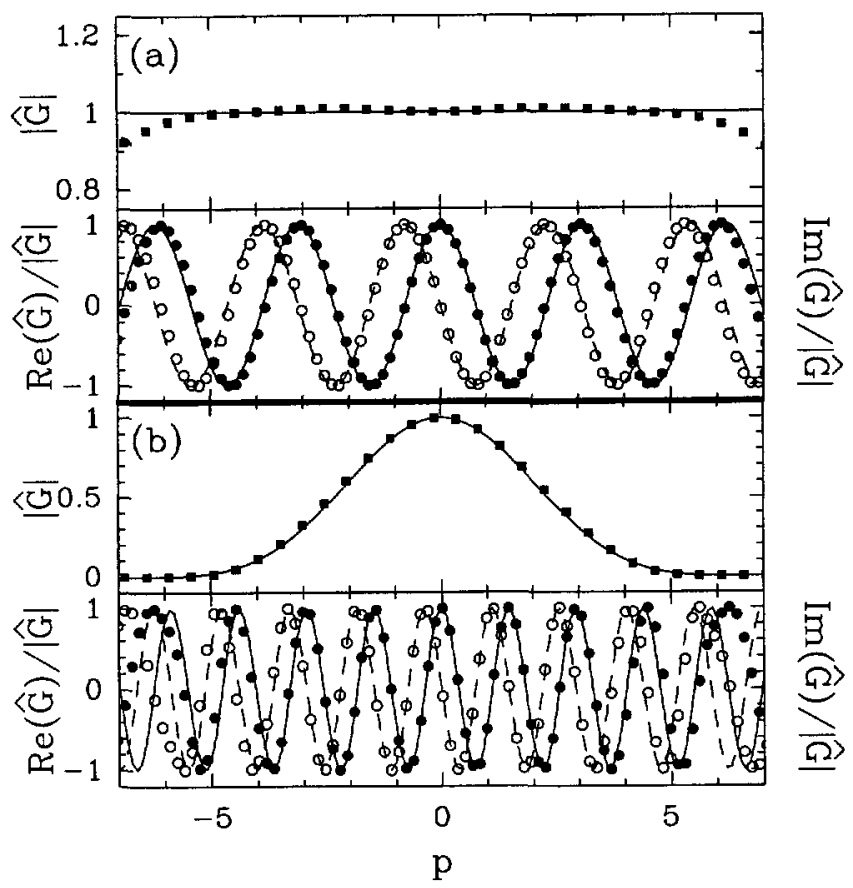

Fig. 1. - Numerical computation of $\hat{G}_{a a^{\prime}}(p)$ for (a) Brownian and (b) multiplicative Bernoulli processes: (ם) $|\hat{G}|$, (•) $\operatorname{Re}(\hat{G}) /|\hat{G}|$ and $(0) \operatorname{Im}(\hat{G}) /|\hat{G}|$. The solid and dashed lines represent the corresponding theoretical predictions. $a=2^{5}$ and $a^{\prime}=2^{11}$ and $\psi=\mathrm{e}^{2 \Omega x}\left(\mathrm{e}^{-x^{2} / 2}-\sqrt{2} \mathrm{e}^{-\Omega^{2} / 4} \mathrm{e}^{-x^{2}}\right)$ is the complex Morlet wavelet [12]. Similar results are obtained with other analyzing wavelets, e.g. the successive derivatives of the Gaussian function

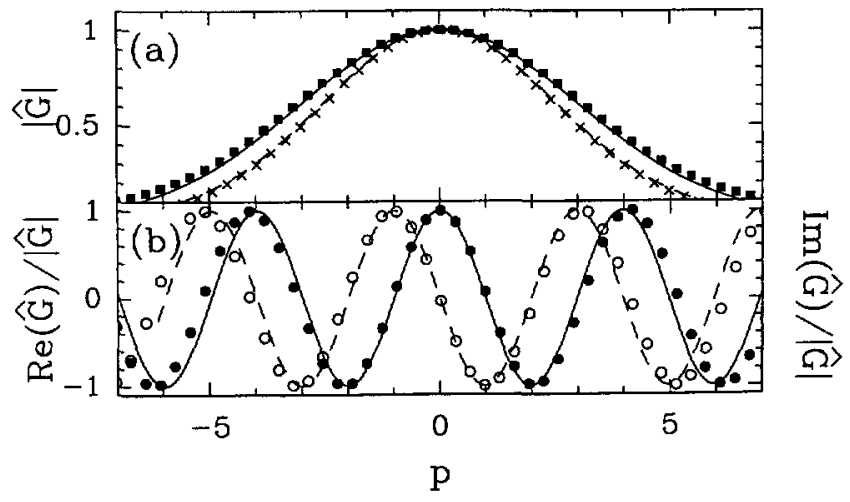

Fig. 2. - Numerical computation of $\hat{G}_{a a^{\prime}}(p)$ for the log-normal model $\left(m=-0.37\right.$ and $\sigma^{2}=0.026$ ): (a) $|\hat{G}|,(\bullet) \operatorname{Re}(\hat{G}) /|\hat{G}|$ and $(\bigcirc) \operatorname{Im}(\hat{G}) /|\hat{G}|$. In (a) the symbols $(\times)$ correspond to results obtained for the log-Poisson model $\left(\lambda=2, \beta=(2 / 3)^{1 / 3}\right.$ and $\left.\gamma=-1 / 9\right)$. The solid and dashed lines represent the corresponding theoretical predictions. $a=2^{5}$ and $a^{\prime}=2^{11}$ Same analyzing wavelet as in Figure 1. 

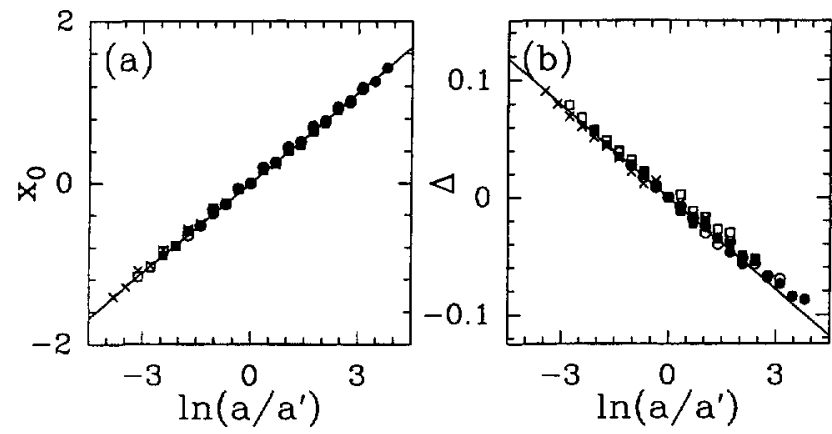

Fig. 3. - Numerical computation of $\hat{G}_{a a^{\prime}}(p)$ for the the log-normal model. (a) $x_{0}\left(a, a^{\prime}\right)=$ $\partial \operatorname{Im}\left(\hat{G}_{a a^{\prime}}\right) /\left.\partial p\right|_{p=0}$ vs. $\ln \left(a / a^{\prime}\right) ;(\mathrm{b}) \Delta\left(a, a^{\prime}\right)=-\partial^{2} \ln \left(\left|\hat{G}_{a a^{\prime}}\right|\right) /\left.\partial p^{2}\right|_{p=0}$ vs. $\ln \left(a / a^{\prime}\right): a^{\prime}=2^{6}$ (•), $2^{7}(\bigcirc), 2^{8}(\mathbf{\square}), 2^{9}(\square)$ and $2^{10}(x)$. The solid lines represent the theoretical predictions: $x_{0}=-m \ln \left(a / a^{\prime}\right)$ and $\Delta\left(a, a^{\prime}\right)=-\sigma^{2} \ln \left(a / a^{\prime}\right)$.

cascade (on a WT orthornormal basis using $L^{1}$ normalization) for parameter values as estimated from turbulence data [9]: $\hat{G}_{a a^{\prime}}(p)=\mathrm{e}^{-\imath p m \ln \left(a / a^{\prime}\right)} \mathrm{e}^{p^{2} \frac{\sigma^{2}}{2} \ln \left(a / a^{\prime}\right)}$ with $m=-0.37$ and $\sigma^{2}=0.026$ for the log-normal model and $\hat{G}_{a a^{\prime}}(p)=\mathrm{e}^{\ln \left(a / a^{\prime}\right)(\lambda(1-\cos (p \ln \beta))-\imath(p \gamma+\lambda \sin (p \ln \beta)))}=$ with $\lambda=2, \beta=(2 / 3)^{1 / 3}$ and $\gamma=-1 / 9$ for the log-Poisson model. Let us note that if $|p \ln \beta| \ll 1$, the $\log$-Poisson process reduces to its quadratic approximation and thus to a log-normal model. For both models one can see in Figure 2 that the numerical data are in perfect agreement with the above analytical expressions. In Figure 3, we have plotted, for the log-normal model and different couples of scales $\left(a, a^{\prime}\right), x_{0}\left(a, a^{\prime}\right)=\partial \operatorname{Im}\left(\hat{G}_{a a^{\prime}}\right) /\left.\partial p\right|_{p=0}$ and $\Delta\left(a, a^{\prime}\right)=-\partial^{2}\left(\ln \left|\hat{G}_{a a^{\prime}}\right|\right) /\left.\partial p^{2}\right|_{p=0}$ as functions of $\ln \left(a / a^{\prime}\right)$. One can see that all the points fall on a unique straight line which matches the theoretical predictions and confirms the scalesimilarity of the cascade.

Let us now proceed to the analysis of fully developed turbulence data. The experimental data were recorded by Gagne and his collaborators $[10 \mathrm{a}, \mathrm{b}]$ in two different flows and represent temporal fluctuations of the longitudinal velocity component. In all our computations we use Taylor hypothesis to identify temporal and spatial variations. The first set of data was recorded at the O.N.E.R.A. wind tunnel in Modane; the Taylor scale based Reynolds number is $R_{\lambda} \simeq 3050$. The second flow is a laboratory jet flow for which $R_{\lambda}=835$. Our sample represents a statistics of $1.5 \times 10^{7}$ points with resolution $\Delta x \simeq 1.2 \eta$ for the wind tunnel data and of about $2.1 \times 10^{7}$ points with resolution $\Delta x \simeq 2 \eta$ for the jet, where $\eta$ is the corresponding dissipative scale. In Figure 4 are represented the modulus and the phase of the function $\hat{G}_{a a^{\prime}}$ that we numerically estimate in the inertial range for both flows $\left(a=2^{5}\right.$ and $a^{\prime}=2^{11}$ ). One can see that these data are very well-fitted by a log-normal model. Actually, we have checked that for all couple of scales $\left(a, a^{\prime}\right)$ in the inertial range, cumulants of $G_{a a^{\prime}}$ of order higher than 2 are negligible. It is clear that, with the available statistical sample, the various log-infinitely divisible cascade models [8-10] cannot be distinguished from their log-normal approximations. In order to test the scale-similarity of turbulence, we reproduce in Figure $5 \mathrm{a}$ the same analysis as in Figure 3a. It is striking (in particular for the jet flow) that the curves obtained for the different scales $\left(a, a^{\prime}\right)$ do not merge on the same straight line. The function $s\left(a, a^{\prime}\right)$ is thus significantly different from $\ln \left(a / a^{\prime}\right)$. In Figure $5 \mathrm{~b}$, the same data are plotted versus $s\left(a, a^{\prime}\right)=\frac{-1}{\alpha}\left(a^{-\alpha}-a^{\prime-\alpha}\right)$ with $\alpha \simeq 0.08$ for the wind tunnel flow and $\alpha \simeq 0.20$ for the jet flow (let us note that in the limit $\alpha \rightarrow 0$, this behavior reduces to $\ln \left(a / a^{\prime}\right)$, a regime which is 


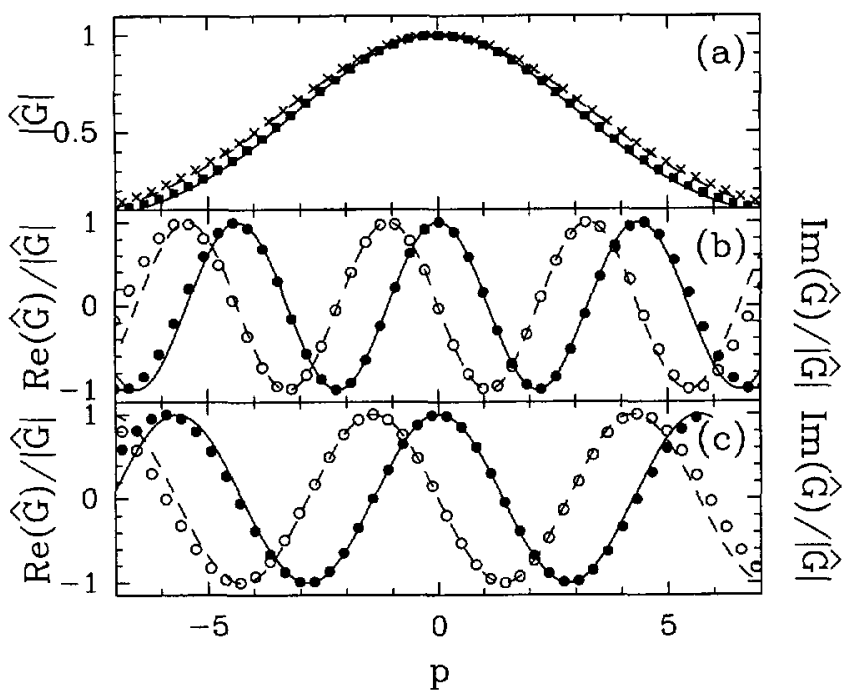

Fig. 4. - (a) $\hat{G}_{a a^{\prime}}(p)$ extracted from wind tunnel (ם) and jet $(\times)$ turbulence experiments. (b) $\operatorname{Re} \hat{G} /|\hat{G}|$ (๑) and $\operatorname{Im} \hat{G} /|\hat{G}|$ (O) for wind tunnel data. (c) Same as (b) for jet data. The solid and dashed lines correspond to the log-normal model after adjusting the parameters $m$ and $\sigma$.
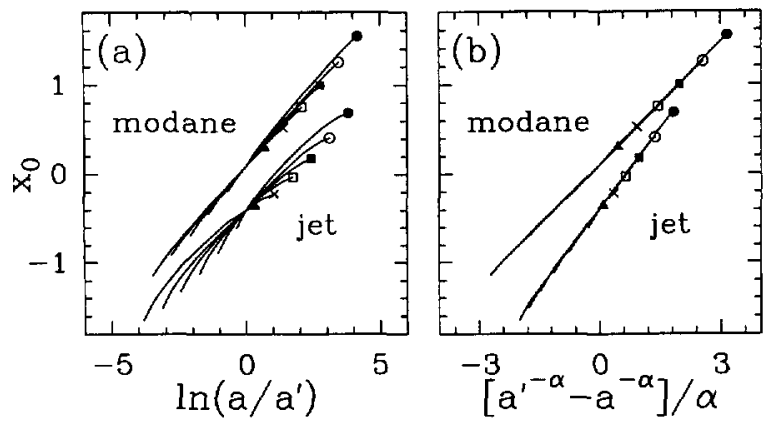

Fig. 5. $-x_{0}\left(a, a^{\prime}\right)=\partial \operatorname{Im}\left(\hat{G}_{a a^{\prime}}\right) /\left.\partial p\right|_{p=0}$ as computed from wind tunnel and jet turbulence experiments. (a) $x_{0}\left(a, a^{\prime}\right)$ vs. $\ln \left(a / a^{\prime}\right)$. (b) $x_{0}\left(a, a^{\prime}\right)$ vs. $\frac{-1}{\alpha}\left(a^{-a}-a^{\prime-\alpha}\right)$ with $\alpha=0.08$ (wind tunnel) and $\alpha=0.20$ (jet) respectively. The different curves correspond to the following values of the reference scale: $a^{\prime}=2^{6}(\bullet), 2^{7}(0), 2^{8}(\mathbf{\square}), 2^{9}(\square)$ and $2^{10}(x)$.

almost reached in the Modane wind tunnel experiment). In this case, whatever $\left(a, a^{\prime}\right)$, all the data points fall on the same line. The velocity fields we have analyzed are therefore not scalesimilar. We have checked that the variance [17] of $G, \Delta\left(a, a^{\prime}\right)$, behaves like the mean $x_{0}\left(a, a^{\prime}\right)$ with the same exponent $\alpha$. According to these findings one would expect the moments of the WT coefficient pdf (actually the structure functions if the WT corresponds to increments) to be of the functional form (instead of a power law):

$$
S_{p}(\ell)=C_{p} \mathrm{e}^{-C_{\psi}^{\alpha} \frac{p-\alpha}{\alpha} \zeta_{p}}
$$

where $C_{p}$ depends on $p, \alpha$ and the analyzing wavelet $\psi, C_{\psi}$ depends on $\psi$, and $\zeta_{p}=-m p-$ $\sigma^{2} p^{2} / 2$ is the quadratic spectrum of the log-normal model. According to this equation, if one 
wants to compare experimental results for different flows and different analyzing wavelets, one can apply the concept of extended self-similarity [18] which consists in estimating the ratio $\zeta_{p} / \zeta_{q}$ by plotting one structure function against the other.

To conclude, we have shown that the notion of abstract cascade inspired by the work of Castaing et al. [10] provides a generalization of the classical cascade models. It does not refer a priori to a particular construction scheme and is characterized by a kernel $G$ which turns out to be computable by a deconvolution method. Application of these notions to fully developed turbulence shows that all the models introduced to fit experimental data can be replaced by their log-normal approximation. Moreover we have reported evidences that fully developed turbulence is not scale-similar but possesses more complex self-similarity properties that are likely to depend on the Reynolds number. These results strongly support the idea of Castaing's group [10] and of a recent work by Dubrulle [16b]. More specific applications to turbulence, e.g. the evolution of the exponent $\alpha$ with the Reynolds number, will be published elsewhere.

\section{Acknowledgments}

We are very grateful to $Y$. Gagne for the permission to use his experimental turbulence signals. This work was supported by the "Direction des Recherches Études et Techniques" under contract (DRET n ${ }^{\circ}$ 95/111).

\section{References}

[1] Monin A.S and Yaglom A.M., Statistical Fluid Mechanics, Vol. 2 (MIT press, Cambridge, MA, 1975).

[2] Frisch U., Turbulence (Cambridge University Press, Cambridge, 1995) and references therein.

[3] Meneveau C. and Sreenivasan K.R., J. Fluid Mech. 224 (1991) 429 and references therein.

[4] (a) Kolmogorov A.N., J. Fluid Mech. 13 (1962) 82; (b) Obukhov A.M., J. Fluid Mech. 13 (1962) 77.

[5] Arneodo A., et al., Europhys. Lett. 34 (1996) 411.

[6] Kolmogorov A.N., C.R. Acad. Sci. USSR 30 (1941) 301.

[7] Mandelbrot B.B., J. Fluid Mech. 62 (1974) 331.

[8] Novikov E.A., Phys. Fluids A 2 (1990) 814; Phys. Rev. E 50 (1995) 3303.

[9] (a) She Z.S. and Lévêque E., Phys. Rev. Lett. 72 (1994) 336; (b) She Z.S. and Waymire E.C., Phys. Rev. Lett. 74 (1995) 262.

[10] (a) Castaing B., Gagne Y. and Hopfinger E.J., Physica D 46 (1990) 177; (b) Gagne Y., Marchand M. and Castaing B., J. Phys. II France 4 (1994) 1; (c) Naert A., Puech L., Chabaud B., Peinke J., Castaing B. and Hebral B., J. Phys. II France 4 (1994) 215;

(d) Castaing B. and Dubrulle B., J. Phys. II France 5 (1995) 895; (e) Chillà F., Peinke J. and Castaing B., J. Phys. II France 6 (1996) 455.

[11] Mandelbrot B.B., Pure Appl. Geoph. 131 (1989) 5.

[12] (a) Meyer Y., Ondelettes (Hermann, Paris, 1990); (b) Daubechies I., Ten Lectures on Wavelets (S.I.A.M., Philadelphia, 1992).

[13] (a) Muzy J.F., Bacry E. and Arneodo A., Int. J. Bifurcation and Chaos 4 (1994) 245;

(b) Arneodo A., Bacry E. and Muzy J.F., Physica A 213 (1995) 232. 
[14] Mallat S. and Zhong S., I.E.E.E. Trans Pattern Anal. Machine Intell. 14 (1992) 710.

[15] Benzi R., Biferale L., Crisanti A., Paladin G., Vergassola M. and Vulpiani A., Physica $D$ 65 (1993) 352.

[16] (a) Dubrulle B., Phys. Rev. Lett. 73 (1994) 959; (b) J. Phys. II France 6 (1996) 1825.

[17] Let us note that $\Delta(a, L)$ is called $\lambda^{2}$ in reference [10] but is computed using a different method.

[18] Benzi R., Ciliberto S., Trippiccione R., Baudet C. and Succi S., Phys. Rev. E 48 (1993) R29. 